\title{
Robustness analysis of a class of decentralized control systems $\dagger$
}

\section{OLE A. SOLHEIM $\ddagger$}

Keywords: robustness; decentralized control; decentralized estimation; singular values; block Gerschgorin theorem.

The paper presents a method for analysing the robustness properties of a class of decentralized control systems. Perturbations both in local and in interconnection parameters are dealt with. The method is based on the use of singular values. Several numerical examples are presented to illustrate the method.

\section{Introduction}

The paper deals with decentralized control of interconnected dynamic systems. We assume that the total system consists of $N$ sub-systems described by

$$
\dot{x}_{i}=A_{i i} x_{i}+B_{i} u_{i}+\sum_{\substack{j=1 \\ j \neq i}}^{N} A_{i j} x_{j}, \quad i=1,2, \ldots, N
$$

where $x_{i}$ is an $n_{i}$-dimensional state vector $\left(\sum_{i=1}^{N} n_{i}=n\right)$ and $u_{i}$ an $r_{i}$-dimensional control vector $\left(\sum_{i=1}^{N} r_{i}=r\right)$. We note that each sub-system has its own control input and that the inter-connections are through the states of the other sub-systems.

The total system may be expressed as

$$
\dot{x}=\bar{A} x+H x
$$

where $\bar{A}=\operatorname{diag}\left\{A_{i i}\right\}$ and the inter-connection matrix $H$ is equal to

$$
H=\left[\begin{array}{cccc}
0 & A_{12} & \ldots & A_{1 N} \\
A_{21} & 0 & \ldots & A_{2 N} \\
\ldots \ldots & \ldots & \ldots & \ldots \ldots \\
A_{N 1} & \ldots & \ldots & 0
\end{array}\right]
$$

In the present paper we consider decentralized control laws of the form

$$
u_{i}=G_{i} x_{i}
$$

There may be several reasons for choosing this structure. In the first place, each control station uses information from, and transmits control signals to, its own sub-system only. This simplifies the signal transmission and also reduces the complexity of the controller. In the second place, the system can be made more robust. In the third place, the computation of the control laws is usually less complex for decentralized schemes.

Received 28 October 1986.

† This paper was presented at the 4 IFAC/IFORS Symposium on Large Scale Systems, Zürich, 26-29 August 1986, and is published with the permission of IFAC.

$\ddagger$ Division of Engineering Cybernetics, The Norwegian Institute of Technology, The University of Trondheim. 7034 Trondheim-NTH, Norway. 
On the other hand, there are some drawbacks concerning overall performance. If, for example, optimal control is considered, the performance of a system with decentralized control will in general be inferior to a system with centralized control. The most serious problem connected to a decentralized control structure is, however, the question of stability of the overall system.

Assuming that the local control systems have been designed with sufficient stability, then it will depend on the inter-connections whether the total system is sufficiently stable or not. A robustness analysis of a decentralized control system will therefore in the first place be concerned with the influence of the inter-connections. In the second place comes the analysis of parameter variations in the local systems. In the present paper both these aspects will be considered.

Based on an LQR (Linear Quadratic Regulator) with $\alpha$-stability (all eigenvalues with real parts less than $-\alpha$ ) a robustness analysis as to the inter-connections is carried out by Sundareshan (1977) and by Darwish, Soliman and Fantin (1979). An analysis based on more general local control systems is presented by Solheim (1980) using the block Gershgorin theorem.

The present paper will be concerned with variations in the inter-connections as well as in the local systems. No specific design of the local controllers is required. We shall, however, for the sake of simplicity, assume real (and of course stable) eigenvalues in the local systems. This may be achieved by a pure modal design or, if a more economic performance is desired, by a combined modal/optimal design (Solheim, 1972). This method will be referred to in the sequel.

The robustness analysis in this paper is based on the singular value concept. A short introduction to the use of singular values in this context is given in $\S 2$.

Section 3 deals with robustness analysis of the local systems, and $\S 4$ with the total system. Simple numerical examples are presented in $\$ 3$ and 4.

\section{Singular values}

Given a complex matrix $A$. The maximum singular value of this matrix is defined as

$$
\bar{\sigma}(A)=\max _{i} \sqrt{ }\left(\lambda_{i}\left(A^{*} A\right)\right)
$$

and the minimum singular value as

$$
\underline{\sigma}(A)=\min _{i} \sqrt{ }\left(\lambda_{i}\left(A^{*} A\right)\right)
$$

where $\lambda_{i}(\cdot)$ denotes an eigenvalue of the argument matrix, and $A^{*}$ is the conjugate transpose of $A$.

Singular values have proved to be of great importance in the analysis and design of robust control systems (MacFarlane (1981), Doyle and Stein (1981), Lehtomaki, Sandell and Athans (1981), Lehtomaki et al. (1981), Lee et al. (1982)). It can, for example, be shown (Lee et al. (1982)) that in a stable feedback system $F$, stability will be maintained as perturbations $\delta F$ are added, provided that

$$
\underline{\sigma}[j \omega \boldsymbol{I}-\boldsymbol{F}]>\overline{\boldsymbol{\sigma}}(\delta \boldsymbol{F})
$$

In general this expression has to be developed as function of the frequency $\omega$. We may, however, simplify the situation considerably if we assume that the feedback system $F$ has real eigenvalues only. 
Assuming real eigenvalues in $F$, we have the relation

$$
\underline{\sigma}(\boldsymbol{F}) \leqslant \underline{\sigma}(j \omega I-F) \quad \text { for all } \omega
$$

One way to see this is to look at the eigenvalue matrix $\Lambda=M^{-1} F M$ where $M$ is an eigenvector matrix of $F$. With $\Lambda$ we get

$$
\sigma(j \omega I-\Lambda)=\min _{i} \sqrt{ }\left(\lambda_{i}^{2}+\omega^{2}\right)
$$

and thus

$$
\underline{\sigma}(\Lambda) \leqslant \underline{\sigma}(j \omega I-\Lambda) \quad \text { for all } \omega .
$$

Since $F$ and $\Lambda$ are similar matrices, we conclude from (9) that (7) also must be valid.

Combining (6) and (7) yields the condition for stability (assuming real eigenvalues in $F$ )

$$
\underline{\underline{\sigma}}(F)>\bar{\sigma}(\delta F)
$$

This is a very useful condition as it permits the study of a large variety of perturbations without too much computational effort. The drawback with this analysis, as with all analysis involving singular values, is the inherent conservatism of the method.

If we instead of considering absolute stability, consider $\alpha$-stability (all eigenvalues to left of $-\alpha$ on the real axis), condition (10) is changed to

$$
\underline{\underline{\sigma}}(F+\alpha I)>\bar{\sigma}(\delta F)
$$

This may also be written

$$
\underline{\sigma}(F)>\bar{\sigma}(\delta F)+\alpha
$$

or

$$
\bar{\sigma}(\delta F)<\underline{\sigma}(F)-\alpha
$$

Using (12) or (13) gives, however, a more conservative result than (11).

\section{Robustness analysis of the local systems}

Consider a local system without interconnections

$$
\begin{aligned}
\dot{x}_{i} & =A_{i i} x_{i}+B_{i} u_{i} \\
y_{i} & =D_{i} x_{i}
\end{aligned}
$$

where $x_{i}, u_{i}$ and $y_{i}$ are $n_{i}$-dimensional state, $r_{i}$-dimensional control and $m_{i}$-dimensional measurement vectors, respectively.

We use the usual quadratic performance index

$$
J_{i}=\frac{1}{2} \int_{0}^{\infty}\left[x_{i}^{T} Q_{i} x_{i}+u_{i}^{T} P_{i} u_{i}\right] d t
$$

where $Q_{i}$ is a symmetric non-negative definite matrix and $P_{i}$ is a symmetric positive definite matrix.

Let us in the first place assume that all state variables are directly accessible. We therefore set $y_{i}=x_{i}$, that is, the measurement matrix $D_{i}=I$. In addition to that the 
controller shall minimize the performance index, we also require that the closedloop system

$$
\dot{x}_{i}=\left(A_{i i}+B_{i} G_{i} D_{i}\right) x_{i}=F_{i} x_{i}
$$

based on the control law

$$
u_{i}=G_{i} y_{i}=G_{i} D_{i} x_{i}
$$

attains prescribed eigenvalues.

In order to achieve this, the weighting matrix $Q_{i}$, for the state, cannot be freely chosen, but must be determined so that the prescribed eigenvalues are obtained (Solheim, 1972; 1979).

Let us consider the following perturbations:

(i) Change $\delta A_{i i}$ in the process matrix $A_{i i}$ due to process parameter variations.

(ii) Change $\delta B_{i}$ in the control matrix $B_{i}$ due to variations in process and actuator parameters.

(ii) Change $\delta D_{i}$ in the measurement matrix $D_{i}$ due to sensor parameter variations.

The total change $\delta F_{i}$ in the closed-loop matrix $F_{i}$ becomes (with $D_{i}=I$ )

$$
\delta F_{i}=\delta A_{i i}+\delta B_{i} G_{i}+B_{i} G_{i} \delta D_{i}
$$

In the present paper we shall consider additive perturbations only.

The purpose of the robustness analysis is now to determine the size of the perturbations that can be tolerated without endangering the stability of the system. Or, in other words, how a given change in the parameters influences the eigenvalues of the system.

Before we go into a detailed discussion of this problem let us also consider the case where all states are not directly measurable. In this case a state estimator has to be included in the controller.

Let $\hat{x}_{i}$ represent the estimated state. With the control law

$$
u_{i}=G_{i} \hat{x}_{i}
$$

we get the total closed-loop system

$$
\left[\begin{array}{c}
\dot{x}_{i} \\
\hat{\dot{x}}_{i}
\end{array}\right]=\left[\begin{array}{c:c}
A_{i i} & B_{i} G_{i} \\
\hdashline K_{i} D_{i} & A_{i i}+B_{i} G_{i}-K_{i} D_{i}
\end{array}\right]\left[\begin{array}{l}
x_{i} \\
\hat{x}_{i}
\end{array}\right]=\hat{F}_{i}\left[\begin{array}{l}
x_{i} \\
\hat{x}_{i}
\end{array}\right]
$$

where $K_{i}$ is the estimator gain.

Assuming changes in $A_{i i}, B_{i}$ and $D_{i}$ of the process (no changes in the model used in the estimator), the changes in $\hat{F}_{i}$ becomes

$$
\delta \hat{F}=\left[\begin{array}{c:c}
\delta A_{i i} & \delta B_{i} G_{i} \\
\hdashline K_{i} \delta D_{i} & 0
\end{array}\right]
$$

The feedback system and the estimator may be designed separately. In the same way as we may design an optimal feedback system with prescribed eigenvalues, we may also design an optimal estimator with prescribed eigenvalues (Solheim, 1972).

We present now two examples to illustrate the use of singular values in the robustness analysis of the local systems. We use the same process for both examples, but in Example 2 a state estimator is included. 


\section{Example 1}

Given the system

$$
A_{i i}=\left[\begin{array}{rr}
-2 & 0 \\
1 & -1
\end{array}\right], \quad B_{i}=\left[\begin{array}{ll}
1 & 0 \\
0 & 1
\end{array}\right], \quad P_{i}=\left[\begin{array}{ll}
1 & 0 \\
0 & 5
\end{array}\right], \quad D_{i}=\left[\begin{array}{ll}
1 & 0 \\
0 & 1
\end{array}\right] .
$$

The eigenvalues of the closed-loop system are specified as $-5,-8$. Using an LQR this is obtained with

$$
Q_{i}=\left[\begin{array}{rr}
62 \cdot 2 & 15 \cdot 6 \\
15 \cdot 6 & 109 \cdot 0
\end{array}\right], \quad G_{i}=\left[\begin{array}{ll}
-6 \cdot 37 & -2 \cdot 6 \\
-0 \cdot 52 & -3 \cdot 63
\end{array}\right]
$$

We compute

$$
\underline{\sigma}\left(F_{i}\right)=\underline{\sigma}\left(A_{i i}+B_{i} G_{i}\right)=4 \cdot 53
$$

The eigenvalues of $F_{i}$ are -5 and -8 . Specifying $\alpha=4$, we would like to know how large variations in the element of $A_{i i}$ that can be tolerated. We choose

$$
\begin{gathered}
\delta A_{i i}=\left[\begin{array}{cc}
\varepsilon_{11} & 0 \\
\varepsilon_{21} & \varepsilon_{22}
\end{array}\right] \\
\bar{\sigma}\left(\delta F_{i}\right)=\bar{\sigma}\left(\delta A_{i i}\right) \leqslant\left|\varepsilon_{11}\right|+\left|\varepsilon_{21}\right|+\left|\varepsilon_{22}\right|
\end{gathered}
$$

With $\alpha=4$ we get

$$
\underline{\sigma}\left(F_{i}+4 I\right)=0.79
$$

Using (11) we get the bounds on the variations

$$
\left|\varepsilon_{11}\right|+\left|\varepsilon_{21}\right|+\left|\varepsilon_{22}\right|<0.79
$$

For the analysis of a system with state estimator we use eqns. (20) and (21) and condition (10)

$$
\underline{\sigma}\left(\hat{F}_{i}\right)>\bar{\sigma}\left(\delta \hat{F}_{i}\right)
$$

\section{Example 2}

We use the same process as in Example 1, but change the measurement matrix $D_{i}$ to

$$
D_{i}=\left[\begin{array}{ll}
0 & 1
\end{array}\right]
$$

We now need a state estimator. We specify the eigenvalues of the estimator: -10 , -12 . This is obtained with the estimator gain

$$
K_{i}=\left[\begin{array}{l}
80 \\
19
\end{array}\right] \text {. }
$$

The feedback matrix $G_{i}$ is the same as in Example 1.

With the same $\delta A_{i i}$ as in Example 1 we get

$$
\bar{\sigma}\left(\delta \hat{F}_{i}\right)=\bar{\sigma}\left(\delta A_{i i}\right) \leqslant\left|\varepsilon_{11}\right|+\left|\varepsilon_{21}\right|+\left|\varepsilon_{22}\right|
$$

With $\alpha=4$

$$
\underline{\sigma}\left(\hat{F}_{i}+4 I\right)=0 \cdot 23
$$


giving the bounds on the variations in this case

$$
\left|\varepsilon_{11}\right|+\left|\varepsilon_{21}\right|+\left|\varepsilon_{22}\right|<0.23
$$

We note that smaller variations are allowed in this case as compared to the case without estimator.

\section{Robustness analysis of the total system}

Consider the total system with local controllers

$$
\dot{x}=(F+H) x
$$

where

$$
\boldsymbol{F}=\operatorname{diag}\left\{\boldsymbol{F}_{\boldsymbol{i}}\right\}
$$

Let us consider two types of perturbations: Perturbations of the local parameters around their nominal values and perturbations of inter-connection parameters around zero.

Neglecting in the first place the local perturbations, we obtain from (10) the condition for a stable system

$$
\underline{\sigma}(F)>\bar{\sigma}(H)
$$

Since

$$
\underline{\sigma}(F)=\min _{i} \underline{\sigma}\left(F_{i}\right)
$$

condition (24) may be expressed as

$$
\min _{i} \underline{\sigma}\left(F_{i}\right)>\bar{\sigma}(H)
$$

This condition reveals the important result that one should try to arrive at a design where no $\sigma\left(F_{i}\right)$ is much smaller than the others.

If $\alpha$-stability of the total system is desired, condition (26) changes to

$$
\underset{i}{\min } \underline{\sigma}\left(F_{i}+\alpha I\right)>\bar{\sigma}(H)
$$

Considering then local perturbations also, condition (24) becomes

$$
\underline{\sigma}(F+\delta F)>\bar{\sigma}(H)
$$

or

$$
\min _{i} \sigma\left(F_{i}+\delta F_{i}\right)>\bar{\sigma}(H)
$$

If $\min _{i} \underline{\sigma}\left(F_{i}\right)$ and $\min _{i} \underline{\sigma}\left(F_{i}+\delta F_{i}\right)$ occur for the same value of the index $i$, then (29) may be expressed as

$$
\min _{i} \underline{\sigma}\left(F_{i}\right)>\bar{\sigma}\left(\delta F_{i}\right)+\bar{\sigma}(H)
$$

When a state estimator is included in the local controllers, we get the system matrix 


$$
\bar{F}=\left[\begin{array}{cccc}
\hat{F}_{1} & \hat{A}_{12} & \ldots & \hat{A}_{1 N} \\
\hat{A}_{21} & \hat{F}_{2} & \ldots & \hat{A}_{2 N} \\
\ldots \ldots & \ldots & \ldots & \ldots \\
\ldots \ldots & \ldots \ldots \ldots & \ldots & \ldots \\
\ldots \hat{A}_{N 1} & \ldots & \ldots & \hat{F}_{N}
\end{array}\right]=\hat{F}+\hat{H}
$$

where

$$
\begin{gathered}
\hat{F}_{i} \text { is given in (20) } \\
\hat{A}_{i j}=\left[\begin{array}{cc}
A_{i j} & 0 \\
0 & 0
\end{array}\right] \\
\hat{F}=\operatorname{diag}\left\{\hat{F}_{i}\right\} \\
\hat{H}=\left[\begin{array}{cccc}
0 & \hat{A}_{12} & \ldots & \hat{A}_{1 N} \\
\hat{A}_{21} & 0 & \ldots & \hat{A}_{2 N} \\
\ldots \ldots \ldots \ldots \ldots \ldots \ldots \\
\ldots \ldots \ldots \ldots \ldots \ldots \ldots \\
\hat{A}_{N 1} & \ldots & \ldots & 0
\end{array}\right]
\end{gathered}
$$

Because of the special form of $\hat{A}_{i j}$ we get

$$
\bar{\sigma}(\hat{H})=\bar{\sigma}(H)
$$

Stability of the system is secured if

$$
\min _{i} \underline{\sigma}\left(\hat{F}_{i}+\delta \hat{F}_{i}\right)>\bar{\sigma}(H)
$$

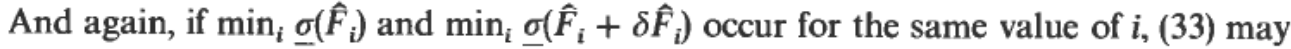
be expressed as

$$
\min _{i} \underline{\sigma}\left(\hat{F}_{i}\right)>\bar{\sigma}\left(\delta \hat{F}_{i}\right)+\bar{\sigma}(H)
$$

To conclude this section let us discuss the special case with only two local systems, that is, $N=2$.

We get

$$
\dot{x}=\left[\begin{array}{cc}
F_{1} & A_{12} \\
A_{21} & F_{2}
\end{array}\right] x=(F+H) x
$$

Thus

$$
\bar{\sigma}(H)=\max \left\{\bar{\sigma}\left(A_{12}\right), \bar{\sigma}\left(A_{21}\right)\right\}
$$

Condition (24) becomes

$$
\min \left\{\underline{\sigma}\left(F_{1}\right), \underline{\sigma}\left(F_{2}\right)\right\}>\max \left\{\bar{\sigma}\left(A_{12}\right), \bar{\sigma}\left(A_{21}\right)\right\}
$$

It is interesting to compare this result with a corresponding result obtained via the block Gerschgorin theorem (Solheim, 1980). In order to do this we diagonalize the local system $F_{i}$ in (35)

$$
\left[\begin{array}{cc}
M_{1}^{-1} & 0 \\
0 & M_{2}^{-1}
\end{array}\right]\left[\begin{array}{cc}
F_{1} & A_{12} \\
A_{21} & F_{2}
\end{array}\right]\left[\begin{array}{cc}
M_{1} & 0 \\
0 & M_{2}
\end{array}\right]=\left[\begin{array}{cc}
\Lambda_{1} & M_{1}^{-1} A_{12} M_{2} \\
M_{2}^{-1} A_{21} M_{1} & \Lambda_{2}
\end{array}\right]
$$


where $M_{1}$ and $M_{2}$ are eigenvector matrices of $F_{1}$ and $F_{2}$ respectively.

Using condition (37) on the system (38) yields

$$
\min \left\{\underline{\sigma}\left(\Lambda_{1}\right), \underline{\sigma}\left(\Lambda_{2}\right)\right\}>\max \left\{\bar{\sigma}\left(M_{1}^{-1} A_{12} M_{2}\right), \bar{\sigma}\left(M_{2}^{-1} A_{21} M_{1}\right)\right\}
$$

Since $\Lambda_{1}$ and $\Lambda_{2}$ are diagonal matrices we have

$$
\min \left\{\underline{\sigma}\left(\Lambda_{1}\right), \underline{\sigma}\left(\Lambda_{2}\right)\right\}=|\lambda|_{\min }
$$

where $|\lambda|_{\min }$ indicates the absolute value of the eigenvalue of

$$
\left[\begin{array}{cc}
\Lambda_{1} & 0 \\
0 & \Lambda_{2}
\end{array}\right]
$$

closest to the imaginary axis. We can thus write (39) as

$$
\max \left\{\bar{\sigma}\left(M_{1}^{-1} A_{12} M_{2}\right), \bar{\sigma}\left(M_{2}^{-1} A_{21} M_{1}\right)\right\}<|\lambda|_{\min }
$$

The block Gerschgorin theorem used on the system (38) states that the inclusive regions for the eigenvalues of the system consist of circles with centres at the eigenvalues of the local systems and with radius equal to $\bar{\sigma}\left(M_{1}^{-1} A_{12} M_{2}\right)$ or $\bar{\sigma}\left(M_{2}^{-1} A_{21} M_{1}\right)$. To have a safe design the largest of these radii must be smaller than the distance from the origin to the nearest eigenvalue. This is exactly the same condition as (40).

We present two examples to illustrate the material presented in this section.

\section{Example 3}

Consider the interconnected system

$$
\left[\begin{array}{l}
\dot{x}_{1} \\
\dot{x}_{2}
\end{array}\right]=\left[\begin{array}{rr:rr}
-2 & 0 & 0 & \gamma_{1} \\
\hdashline 0 & -1 & 0 & 0 \\
\hdashline 0 & \gamma_{2} & -2 & 0 \\
\hdashline & 1 & -1
\end{array}\right]\left[\begin{array}{l}
x_{1} \\
x_{2}
\end{array}\right]+\left[\begin{array}{rr}
1 & 0 \\
0 & 1 \\
\hdashline 1 & 0 \\
0 & 1
\end{array}\right]\left[\begin{array}{l}
u_{1} \\
u_{2}
\end{array}\right]
$$

The local systems are the same as the system in Example 1. We also use the controller in that example for the local systems. The eigenvalues of the closed-loop local system are -5 and -8 .

Assuming the same local perturbations as in Example 1, condition (27) together with (30) yields, when we desire $\alpha$-stability with $\alpha=4$ :

$$
\underline{\sigma}\left(F_{i}+4 I\right)>\bar{\sigma}\left(\delta F_{i}\right)+\bar{\sigma}(H), \quad i=1,2
$$

With numerical values:

$$
\max \left\{\left|\gamma_{1}\right|,\left|\gamma_{2}\right|\right\}+\left|\varepsilon_{11}\right|+\left|\varepsilon_{21}\right|+\left|\varepsilon_{22}\right|<0.79
$$

This condition expresses the combination of perturbation in local and interconnection parameters that can be tolerated to have $\alpha$-stability with $\alpha=4$.

\section{Example 4}

We use the same process as in Example 3, but include a state estimator in the local controllers. These are the same as in Example 2.

With $\alpha=4$ we get from condition (34)

$$
\underline{\sigma}\left(\hat{F}_{i}+4 I\right)<\bar{\sigma}\left(\delta \hat{F}_{i}\right)+\bar{\sigma}(H), \quad i=1,2
$$


With numerical values

$$
\max \left\{\left|\gamma_{1}\right|,\left|\gamma_{2}\right|\right\}+\left|\varepsilon_{11}\right|+\left|\varepsilon_{21}\right|+\left|\varepsilon_{22}\right|<0 \cdot 23
$$

\section{Concluding remarks}

We have presented a method for analysing the robustness properties of a class of decentralized control systems. In order to do this we have also discussed the robustness of the local control systems.

The method is based on the use of singular values. To facilitate the analysis we have assumed that the local closed-loop systems are designed so that they have real (and of course stable) eigenvalues.

\section{REFERENCES}

Darwish, M., Soliman, H. M., and Fantin, J. (1979). Decentralized stabilization of largescale dynamic systems. IEEE Trans. Syst. Man. Cybernet., 9, 717-720.

Doyle, J. C., and SterN, G. (1981). Multivariable feedback design: Concepts for a classical/ modern synthesis. IEEE Trans. Autom. Contr., 26, 4-16.

Lee, W. H., Gully, S. W., Eterno, J. S., and Sandell, N. R. (1982). Structural information in robustness analysis. American Control Conference (ACC), Arlington, Va, June 14-16, 1982.

Lehtomaki, N. A., Castanon, D., Levy, B., Stein, G., Sandell, N. R., and Athans, M. (1981). Robustness tests utilizing the structure of modelling errors. 20th CDC, San Diego, Dec. 16-18, 1981.

MacFARLANE, A. G. J. (1981). Characteristic and principal gains and phases and their use as multivariable control design tools. AGARD Lecture series No. II7, Paper No. 2, 1-34.

SolheIM, O. A. (1972). Design of optimal control systems with prescribed eigenvalues. Int. $J$. Control, 15, 142-160.

Solheim, O. A. (1979). Design of a class of optimal controller via low-order Riccati equations. IF AC Symposium on Computer Aided Design of Control Systems. Zürich, Aug. 29-31, 1979.

Solheim, O. A. (1980). On the use of a block analogue of the Gerschgorin circle theorem in the design of decentralized control of large-scale systems. IF AC Symposium on Large Scale Systems, Toulouse, June 24-26, 1980.

Sundareshan, M. K. (1977). Exponential stabilization of large-scale systems. Decentralized and multilevel schemes. IEEE Trans. Syst. Man. Cybernet., 9, 717-720. 